\title{
Modelo del citoma como biomarcador de la severidad de la preeclampsia.
}

Cytoma model as a biomarker of preeclampsia severity.

\author{
A. Hernández-Ceruelos ${ }^{a}, S$. Muñoz-Juárez ${ }^{b}$, L. López-Contreras $^{c}$, S. Sauz-Hernández ${ }^{d}$, G. \\ Lara-Blanco $^{e}$, J. Silva-Lozada $^{f}$
}

\begin{abstract}
:
Preeclampsia is a hypertensive syndrome typical of pregnancy, which has multisystem repercussions and represents an important factor of maternal morbidity and mortality worldwide. The objective of this study was to determine the genetic damage that this pathology causes in human cells, using the cytoma model as a biomarker to assess samples of oral epithelium. Our results show an increase in chromosomal alterations in the cases of patients diagnosed with preeclampsia compared to controls, highlighting the increase in the frequency of binucleated cells and cells in a state of apoptosis.
\end{abstract}

Keywords:

Preeclampsia, cytoma, genetic damage, binucleated, apoptosis

\section{Resumen:}

La preeclampsia es un síndrome hipertensivo propio del embarazo, el cual tiene repercusiones multisistémicas y representa a nivel mundial un importante factor de morbilidad y mortalidad materna. El objetivo de este estudio fue determinar el daño genético que esta patología ocasiona en las células humanas, usando como biomarcador el modelo del citoma para valorar muestras de epitelio oral. Nuestros resultados evidencian un incremento de alteraciones cromosómicas en los casos de pacientes diagnosticados con preeclampsia en comparación con los controles, destacando el aumento en la frecuencia de células binucleadas y de células en estado de apoptosis.

\section{Palabras Clave:}

Preeclampsia, citoma, daño genético, binucleadas, apoptosis

\section{Introducción}

Alrededor del $6-8 \%$ de los embarazos, a nivel mundial, muestran complicaciones debido a preeclampsia (PE), y se estima que esta patología, junto con el resto de estados hipertensivos del embarazo (EHE), representan el $26 \%$ de mortalidad en América Latina y el Caribe (Steegers et al. 2010), en México según el COMEGO (Colegio Mexicano de Especialistas en Ginecología y Obstetricia) la PE ocasiona el $22-23 \%$ de las muertes maternas en el país y de 50,000 a 60, 000 muertes anuales en todo el mundo (2014).

PE es un trastorno del embarazo caracterizado por hipertensión y proteinuria, de nueva aparición, posterior a la vigésima semana de gestación. Sin embargo, otros

\footnotetext{
a Autor de Correspondencia, Instituto de Ciencias de la Salud, Área Académica de Medicina, Cuerpo Académico de Salud Pública, Universidad Autónoma del Estado de Hidalgo, https://orcid.org/0000-0003-4065-4589, Email: alejandra.ceruelos@ gmail.com

b Departamento de Investigación, Hospital General de Pachuca, Hidalgo. https://orcid.org/0000-0001-5118-9024 Email: sergiomzjz@gmail.com

c Instituto de Ciencias de la Salud, Área Académica de Medicina, Universidad Autónoma del Estado de Hidalgo, https://orcid.org/0000-00018502-2310, Email: luilli_801@yahoo.com.mx

${ }^{\mathrm{d}}$ Instituto de Ciencias de la Salud, Área Académica de Medicina, Universidad Autónoma del Estado de Hidalgo, https://orcid.org/0000-00033336-1649, Email: sgsauz16@ hotmail.com

Servicio de Ginecología y Obstetricia, Hospital General de Pachuca, Hidalgo. https://orcid.org/0000-0002-7084-8889, Email: guillermolarablanco@gmail.com

${ }_{\mathrm{f}}^{\mathrm{f}}$ Instituto de Ciencias de la Salud, Universidad Autónoma del Estado de Hidalgo, Médico Pasante del Servicio Social, https://orcid.org/00000003-1353-1030, Email: jarimsilva@gmail.com
} 
signos y síntomas pueden estar presentes al momento del diagnóstico, en ausencia de proteinuria, en algunas mujeres (ACOG, 2019).

En el diagnóstico de los EHE las guías internacionales o de cada país no muestran variabilidad importante. La Guía de Manejo Clínico de la ACOG en su actualización del 2019, define los criterios diagnósticos para PE (Tabla 1). También identifica datos de severidad para la PE, los cuales incrementan el riesgo de morbilidad y mortalidad en estas pacientes: Presión sistólica mayor o igual a 160 $\mathrm{mmHg}$ o diastólica mayor o igual a $110 \mathrm{mmHg}$ en dos tomas separadas por al menos 4 horas, trombocitopenia $<100,000 \times 109 / L$, injuria hepática manifestada por enzimas hepáticas aumentadas (al doble de sus valores normales) y dolor en hipocondrio derecho o epigastrio, insuficiencia renal (Creatinina $>1.1 \mathrm{mg} / \mathrm{dL}$ o el doble de su valor inicial, en ausencia de otra nefropatía), edema pulmonar, cefalea de recién aparición que no responde a tratamiento médico y no se identifica otro diagnóstico para ella o alteraciones visuales.

\begin{tabular}{|c|c|c|}
\hline Criterio & & Comentario \\
\hline $\begin{array}{l}\text { Presión } \\
\text { arterial }\end{array}$ & $\begin{array}{l}\text { Mayor o igual a } \\
140 / 90 \mathrm{~mm} \mathrm{Hg}\end{array}$ & $\begin{array}{l}\text { Dos ocasiones } \\
\text { separadas en al } \\
\text { menos } 4 \text { horas, } \\
\text { posterior a la } \\
\text { vigésima } \\
\text { semana de } \\
\text { gestación en } \\
\text { una mujer con } \\
\text { presión arterial } \\
\text { normal } \\
\text { previamente. }\end{array}$ \\
\hline Proteinuria & $\begin{array}{l}300 \mathrm{mg} \text { en } 24 \\
\text { horas; o } \\
\text { Proteína/Creatinina } \\
\text { igual o mayor de } \\
0.3 \mathrm{mg} / \mathrm{dL} \text {; o tira } \\
\text { reactiva de } 2+{ }^{*}\end{array}$ & $\begin{array}{l}\text { En ausencia de } \\
\text { proteinuria: } \\
\text { Trombocitopenia } \\
(100,000 \\
\left.10^{9} / \mathrm{L}\right), \\
\text { Creatinina >1.1 } \\
\text { mg/dL o el doble } \\
\text { de su valor } \\
\text { inicial, elevación } \\
\text { de al } \\
\text { transaminasas } \\
\text { hepáticas } \\
\text { doble del valor } \\
\text { normal, edema } \\
\text { pulmonar, } \\
\text { cefalea de } \\
\text { recién aparición } \\
\text { que no responde } \\
\text { a tratamiento } \\
\text { médico y no se }\end{array}$ \\
\hline
\end{tabular}

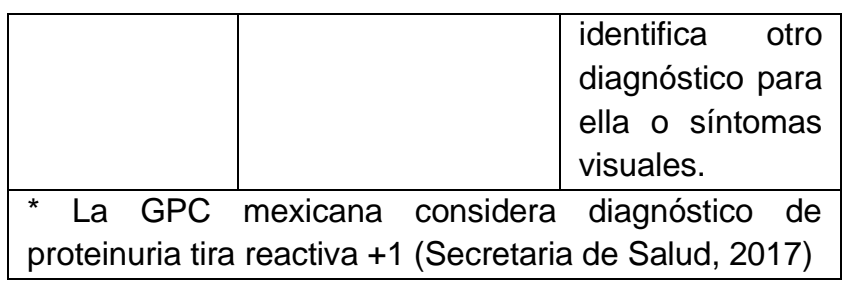

Tabla 1. Criterios diagnósticos de preeclampsia (ACOG, 2019).

EI COMEGO (2014) realiza las siguientes consideraciones respecto a los estados hipertensivos del embarazo y los clasifica en:

- Hipertensión gestacional: Cifras tensionales mayor o igual a $140 / 90 \mathrm{mmHg}$, posterior a la vigésima semana de gestación y en ausencia de proteinuria.

- Hipertensión crónica: Cuando se realizó el diagnostico en el periodo pregestacional, ocurre antes de la semana 20 de gestación o persiste posterior al puerperio.

- Hipertensión crónica con preeclampsia concomitante: Pacientes con diagnóstico previo de hipertensión crónica con: 1) elevación súbita de cifras tensionales o necesidad de aumentar dosis de medicamentos antihipertensivos en pacientes controladas, 2) proteinuria o aumento de la misma si ya existía, 3) datos de severidad.

- Eclampsia: Presentación de convulsiones tónico-clónicas generalizadas en pacientes preeclampticas, sin alteración neurológica previa. Puede ocurrir antes, durante o después del parto.

- Síndrome de HELLP (Por sus siglas en inglés: Hemolysis, elevated liver enzymes, low platelet count): Se caracteriza por datos de hemolisis (elevación de Bilirrubina indirecta $>1.2$ mg, alteración en frotis sanguíneo), elevación de enzimas hepáticas al doble de su valor normal (AST >70, ALT >70) y trombocitopenia $\left(<100,000 \times 10^{9} / L\right)$.

Al considerarse la preeclampsia un síndrome multifactorial, aún no se tiene una fisiopatología que permita explicar el desarrollo de dicha patología; sin embargo se han desarrollado algunas teorías: una implantación anormal con el resultado de hipoxia placentaria y la hipoxia de reperfusión, lo cual resulta en daño al sincitiotrofoblasto y restricción del crecimiento fetal. Dentro de la implantación anormal influyen mecanismos secundarios que incluyen un desbalance entre factores proangiogénicos y antigiogénicos, estrés oxidativo materno y disfunción endotelial. (Gómez Carbajal, 2014)

El daño al endotelio vascular es uno de los eventos patológicos primarios en la preeclampsia. Este daño esta mediado por un incremento en el estrés oxidativo por la 
proliferación de radicales libres de oxígeno (ROS), los cuales son particularmente dañinos para el material genético en donde el daño al DNA, lo que se asocia con varias condiciones patológicas como cáncer, enfermedades cardiacas y neurodegenerativas, inflamación y envejecimiento (Hilali et al., 2013).

Se ha encontrado una concentración incrementada de muchos marcadores de estrés oxidativo en la preeclampsia, como los peroxinitritos. La concentración de peroxinitritos en el endotelio vascular es mucho más elevada en mujeres con preeclampsia que en aquellas con embarazos normales, lo que produce alteraciones a nivel endotelial con aumento en vasoconstricción y disminución de riego sanguíneo; lo que condiciona la hipoxia placentaria. Al presentar un aumento de estrés oxidativo a nivel de retículo endoplásmico en mujeres con preeclampsia, se activa un sistema homeostático; sin embargo, si este sistema falla, se activan las vías apoptóticas que alteran la función placentaria, como consecuencia de estos procesos durante el segundo y el tercer trimestre de embarazo puede condicionar la restricción del crecimiento intrauterino. (Gómez Carbajal, 2014).

Las consecuencias de las interacciones entre los mutágenos o condiciones adversas en un organismo como ocurre con el estrés oxidativo, que pueden reaccionar con el DNA provocando la formación de aductos, sitios álcali lábiles, rupturas de cadena, provocando ya sea cambios en la secuencia de los nucleótidos como aberraciones tanto numéricas como estructurales. El destino final de la célula está determinado por la variedad de las lesiones, y la capacidad de ser reparadas o eliminadas por apoptosis (Decordier et al., 2007).

Los micronúcleos se pueden medir en linfocitos, células mucosa oral, nasal, esofágica, bronquial, de vejiga urinaria y cérvix uterino. Los micronúcleos en células de exfoliación emergen durante la mitosis de las capas basales del epitelio como DNA extracromosómico provocado por la acción de clastógenos es decir agentes que causen rupturas cromosómicas o bien aneunógenos, los cuales alteran el uso acromático provocando perdida de cromosomas completos.

No hay diferencia en la frecuencia de micronúcleos cuando los individuos se exponen a los agentes mutagénicos en un periodo de años o por un corto tiempo (Majer et al., 2001).

Un estudio utilizando el modelo de micronúcleos en cultivo de linfocitos demostró un incremento de ellos en mujeres con preeclampsia al compararse con mujeres cuyos embarazos fueron normales (Furness et al., 2010).
Esta técnica citogenética se puede aplicar para establecer el impacto nutricional y del estilo de vida en el daño al DNA, así como la tasa de proliferación y muerte celular, en donde además de la expresión de los micronúcleos se determina la presencia de anormalidades nucleares como medida de daño genotóxico, efecto citoestático o citotóxico (Bolognesi et al., 2013).

El objetivo del presente trabajo fue determinar la asociación entre la preeclampsia-eclampsia y el modelo del citoma en epitelio oral como biomarcador de daño genotóxico y muerte celular provocados por la patología.

\section{Material y métodos.}

El diseño realizado fue a base de casos y controles, en este estudio los casos se consideraron mujeres con preeclamsia y eclampsia, los controles se conformaron de aquellas mujeres sin la presencia de estas enfermedades. Los estudios de casos y controles se basan en la identificación de los casos en una determinada población durante un periodo de observación definido, se tiene el propósito de estimar la proporción de individuos expuestos y no expuestos identificados una muestra de 42 casos y 42 controles representativa de mujeres embarazadas del hospital general de Pachuca en el periodo Enero- Junio 2018. Se invitó a participar a las pacientes y controles de manera verbal y al momento de aceptar se les hizo firmar un consentimiento informado, indicando que los datos obtenidos se mantuvieron en el anonimato y solo son con fines de la investigación.

Una vez obtenido el consentimiento, se realizó la obtención de información a partir del expediente clínico, de donde se extrajeron los datos clínicos, variables antropométricas y socioeconómicas, así como las relacionadas con el diagnóstico de preeclampsia

Se procedió a tomar la muestra de células epiteliales siguiendo el siguiente procedimiento: Se indicó al sujeto que se enjuagara la boca con $50 \mathrm{ml}$ de solución salina fisiológica (SSF) tibia, posteriormente se solicitó que abriera la boca y con un abatelenguas estéril de madera, previamente humedecido en SSF, se raspó gentilmente, pero con firmeza sobre el carrillo interno, tomando suficiente cantidad de células. Inmediatamente se realizó el extendido celular sobre la laminilla que contiene 2 gotas de SSF y se dejó secar al aire.

Se prepararon 2 muestras de cada carrillo. Se fijaron las laminillas en solución de Carnoy por 30 min y se dejaron secar al aire por al menos 24 horas, para continuar con la técnica de tinción de Feulgen. 
Una vez teñida la laminilla, las células epiteliales se distinguen con el núcleo teñido de color magenta y el citoplasma de color verde. Se clasificaron 1000 células epiteliales por paciente de acuerdo a la morfología del núcleo siguiendo los criterios de Bolognesi y colaboradores (2013): morfología nuclear, se determinó si son normales o bien apoptóticas.

1. Célula diferenciada normal.

2. Célula apoptótica con picnocitois (núcleo altamente condensado e hipercromático).

3. Celula apoptótica con condensación de cromatina (núcleo con patrón de tinción rayado).

4. Célula apoptótica con cariorrhexis (núcleo vacuolizado).

5. Célula con cariolisis (sin núcleo).

6. Células micronucleadas.

7. Células binucleadas.

8. Células bulki.

9. Células con puente.

En cuanto al análisis estadístico se utilizó el programa STATA versión 12.0.

A las variables cuantitativas se les calcularon media, desviación estándar y varianza, y posteriormente se comparó el grupo caso contra el grupo control mediante una prueba de t de Student con un valor de $\mathrm{P}<0.05$.

A las variables cualitativas de ambos grupos se les comparó utilizando la prueba de Ji- cuadrada con un nivel de confianza del $95 \%$.

Después del análisis bi-variado se procedió a realizar medidas de asociación como la determinación de razón de momios, y razón de momios ajustada.

\section{Resultados.}

La tabla 2 muestra las características generales de la población de estudio, en donde se puede apreciar que no se tienen diferencias estadísticamente significativas en cuanto a edad, índice de masa corporal ni edad gestacional de las participantes, pero si en las cifras de tensión arterial, tanto sistólica como diastólica.

\begin{tabular}{|l|l|l|l|}
\hline & \multicolumn{3}{|c|}{ GRUPO } \\
\hline & Caso & Control & Total \\
\hline $\mathrm{N}$ & 54 & 52 & 106 \\
\hline Edad X + S.D. & $25.4+$ & $25.6+6.30$ & $25.5+$ \\
& 6.25 & & 6.24 \\
\hline IMC X + S.D. & $27.5+$ & $26.8+2.13$ & $27.4+$ \\
& 3.23 & & 2.78 \\
\hline
\end{tabular}

\begin{tabular}{|l|l|l|l|}
\hline $\begin{array}{l}\text { Edad } \\
\text { gestacional X } \\
\text { + S.D. }\end{array}$ & $\begin{array}{l}36.0+ \\
3.49\end{array}$ & $35.3+6.24$ & $\begin{array}{l}34.7+ \\
5.02\end{array}$ \\
\hline $\begin{array}{l}\text { Tensión } \\
\text { arterial } \\
\text { sistólica X + } \\
\text { S.D. }\end{array}$ & $\begin{array}{l}136.7+ \\
24.8\end{array}$ & $110.1+$ & 12.15 \\
\hline $\begin{array}{l}\text { Tensión } \\
\text { arterial } \\
\text { diastólica X + } \\
\text { S.D. }\end{array}$ & $\begin{array}{l}89.5+ \\
19.3\end{array}$ & $71.0+8.54$ & $80.4+$ \\
\hline
\end{tabular}

Tabla 2. Características generales de las pacientes participantes en el estudio.

Con respecto a los antecedentes ginecológicos, se puede apreciar, el $50 \%$ eran primigestas, $50 \%$ multigestas $(29 \%$ secundigestas, $14 \%$ tercera gesta, $2 \%$ cuarta gesta y $5 \%$ quinta gesta). La mayor parte de las participantes había resuelto sus embarazos previos vía parto vaginal y solo el $8 \%$ de las participantes tenían antecedente de aborto. De las pacientes incluidas en el estudio se presentaron las siguientes comorbilidades: 1 paciente que cursaba con un embarazo gemelar, 1 paciente con antecedente de preeclampsia en embarazo anterior y 2 pacientes con diagnóstico previo al embarazo de diabetes mellitus, sin embargo, ninguna de estas comorbilidades presentó significancia estadística, de importancia, para considerarse un factor de riesgo para desarrollo de algún estado hipertensivo en el embarazo.

Dentro de las pacientes caso, presentaron los siguientes estados hipertensivos: hipertensión gestacional $28 \%$, preeclampsia $10 \%$, preeclampsia con criterios de severidad $52 \%$, síndrome de HELLP $8 \%$ y eclampsia $2 \%$. Se obtuvieron posterior a la lectura de laminillas con la prueba estadística Chi2, los resultados de apoptosis y de anormalidades nucleares, comparando las poblaciones de casos y controles.

Dentro de los cambios celulares compatibles con apoptosis se encontraron: picnosis, células fantasmas, kario y células condensadas. Se obtuvieron valores de células con presencia de cariorrexis, picnosis con valores de $p=0.0177$ y $p=0.0234$ respectivamente, con valores estadísticamente significativos. Así mismo del total de células apoptóticas se obtuvo un valor de $p<0.05$ considerándose estadísticamente significativos en pacientes con presencia de estados hipertensivos, en específico en preeclampsia con criterios de severidad. (Tabla 3).

Dentro de las anormalidades nucleares que se observaron en las muestras con la prueba estadística Chi2, comparando casos y controles; fueron células binucleadas, con valor de $\mathrm{p}=0.49$ estadísticamente 
significativo, contrarios a lo inicialmente propuesto, la presencia de micronúcleos; de los cuales su valor de $\mathrm{P}=0.754$, con valores que no resultan estadísticamente significativos. Así mismo el total de anormalidades nucleares en conjunto obtenidos de las muestras de las pacientes, se obtiene una $\mathrm{P}=0.049$ con valor estadísticamente significativo. (Tabla 4). Para estudiar la probabilidad o riesgo de presentar preeclampsia, se realizó un análisis de regresión logística, tomando como referencia la razón de momios ajustada, con un intervalo de confianza del $95 \%$.

Este análisis se realizó en la población de estudio. Se encontraron como factor protector la primiparidad, conociendo que, a mayor número de gestas, mayor riesgo de desarrollar preeclampsia en embarazos posteriores. Las variables como edad, incluso el sobrepeso se encuentra como factor de riesgo para desarrollo de preeclampsia, con una razón de momios de 1 y 1.2 respectivamente (Tabla 5).

\section{Discusión}

El daño cromosómico puede ser medible de acuerdo con Fenech a base del modelo de Micronúcleos, puede utilizarse la técnica de bloqueo durante el proceso de citocinesis que utiliza citocalasina- $B$, que inhibe la polimerización de actina; permitiendo que dichas células fueran reconocidas por su apariencia binucleada, permitiendo realizar pruebas de genotoxicidad in vitro y corroborar alteraciones celulares, así como pacientes control no expuestos. Estas muestras celulares provenientes de células epiteliales bucales, nasales y uroteliales de individuos sanos; que posteriormente fueron expuestas a genotóxicos y como resultado contienen un gran número de células degeneradas. Esto es medible en dos poblaciones: la primera considerándose sana, y la segunda expuesta a algún factor genotóxico, en esta tesis las pacientes caso se consideran pacientes con algún estado hipertensivo en el embarazo, de distintos grados de severidad. Se consideran estos factores estrés oxidativo. (Fenech et al, 2013)

De acuerdo con la revisión realizada por Toljic, presenta una asociación entre el estrés oxidativo, que es causado por una alteración en la regulación de aumento en la producción y una disminución de eliminación de las especies reactivas del oxígeno, que representan un papel importante en la fisiopatología de la hiperglucemia y la hipertensión. La hiperglucemia aumenta el estrés oxidativo a través de varios mecanismos moleculares, como la autooxidación y la betaoxidación de la glucosa, con el aumento de la producción de especies reactivas de oxígeno en las mitocondrias. La hipertensión arterial también se asocia con una mayor generación de especies reactivas de oxígeno. (Toljic et al 2017)

El estrés oxidativo induce daño a los lípidos de las membranas celulares, proteínas y ADN. Distintos marcadores de inflamación se conocen en la patogenia de la preeclampsia: malondialdehído (MDA), prostaglandinas F2alfa, factor de necrosis tumoral alfa e interleucina 6 IL6. EI MDA se forma por la peroxidación lipídica de ácidos grasos insaturados y es un marcador de la degradación oxidativa de la membrana celular, inducidas por radicales libres, por lo que ha sido ampliamente utilizado como biomarcador del estrés oxidativo. Varios estudios han demostrado que el MDA está elevadas en pacientes con diabetes mellitus e hipertensión arterial. El estrés oxidativo, causa bien conocida de daño en el ADN e inestabilidad del genoma, puede conducir a reordenamientos cromosómicos.

De acuerdo a los resultados obtenidos en relación a micronúcleos, en ambas poblaciones se determinó que no existen diferencias significativas entre ambos grupos, esto puede ser consecuencia: que el tejido epitelial o cantidad de muestra no es suficiente para el análisis; o que no existe daño del tejido por no cursar con la patología hipertensiva (pacientes control). 


\begin{tabular}{|c|c|c|c|c|c|c|c|c|c|c|}
\hline & \multicolumn{2}{|c|}{ Micronúcleos } & \multicolumn{2}{|l|}{ Puente } & \multicolumn{2}{|l|}{ Bulki } & \multicolumn{2}{|c|}{ Binúcleos } & \multicolumn{2}{|c|}{$\begin{array}{l}\text { Anormalidades } \\
\text { nucleares }\end{array}$} \\
\hline & Control & Caso & Control & Caso & Control & Caso & Control & Caso & Control & Caso \\
\hline Media & 1.5 & 1.718 & 0.117 & 0.156 & 0.888 & 0.375 & 4.058 & 7.625 & 5.764 & 9.875 \\
\hline Valor mínimo & 0.359 & 0.898 & 0.025 & 0.005 & 0.043 & 0.048 & 1.787 & 4.719 & 2.989 & 8.540 \\
\hline Valor máximo & 2.640 & 2.539 & 0.260 & 0.317 & 0.220 & 0.701 & 6.329 & 10.530 & 8.540 & 13.193 \\
\hline $\begin{array}{l}\text { Desviación } \\
\text { estándar }\end{array}$ & 3.268 & 2.275 & 0.409 & 0.447 & 0.378 & 0.906 & 6.508 & 8.059 & 7.954 & 9.202 \\
\hline $\mathbf{P}$ & 0.754 & & 0.715 & & 0.095 & & $0.049^{*}$ & & $0.049^{*}$ & \\
\hline
\end{tabular}

Tabla 4. Presencia de anormalidades nucleares en muestras recolectadas en pacientes control y pacientes caso incluidas en estudio realizado en Hospital General de Pachuca durante el periodo Enero-Junio 2016.

Fuente: Expediente clinico

\begin{tabular}{|c|c|c|c|c|c|c|c|c|c|c|c|c|}
\hline & \multicolumn{2}{|c|}{ Normal } & \multicolumn{2}{|c|}{ Condensados } & \multicolumn{2}{|c|}{ Cariorrexis } & \multicolumn{2}{|c|}{ Picnóticos } & \multicolumn{2}{|c|}{ Fantasmas } & \multicolumn{2}{|c|}{ Apoptosis } \\
\hline & $\begin{array}{l}\text { Contr } \\
\text { ol }\end{array}$ & Caso & $\begin{array}{l}\text { Contr } \\
\text { ol }\end{array}$ & Caso & Control & Caso & Control & Caso & $\begin{array}{l}\text { Contr } \\
\text { ol }\end{array}$ & Caso & Control & Caso \\
\hline Media & $\begin{array}{l}316.4 \\
7\end{array}$ & $\begin{array}{l}302.2 \\
5\end{array}$ & 91.97 & $\begin{array}{l}141.0 \\
3\end{array}$ & $\begin{array}{l}571.71 \\
8\end{array}$ & $\begin{array}{l}106.6 \\
2\end{array}$ & 34.76 & 74.65 & 73.41 & $\begin{array}{l}398.4 \\
11\end{array}$ & 198.26 & 249.40 \\
\hline $\begin{array}{l}\text { Valor } \\
\text { mínimo }\end{array}$ & $\begin{array}{l}248.1 \\
1\end{array}$ & $\begin{array}{l}246.7 \\
5\end{array}$ & 61.67 & $\begin{array}{l}113.6 \\
1\end{array}$ & $\begin{array}{l}504.26 \\
4\end{array}$ & 64.20 & 27.25 & 39.43 & 50.46 & $\begin{array}{l}338.5 \\
18\end{array}$ & 160.58 & 204.44 \\
\hline $\begin{array}{l}\text { Valor } \\
\text { máximo }\end{array}$ & $\begin{array}{l}384.8 \\
2\end{array}$ & $\begin{array}{l}357.7 \\
4\end{array}$ & $\begin{array}{l}122.2 \\
6\end{array}$ & $\begin{array}{l}168.4 \\
4\end{array}$ & $\begin{array}{l}639.17 \\
2\end{array}$ & $\begin{array}{l}149.0 \\
4\end{array}$ & 42.27 & $\begin{array}{l}109.8 \\
7\end{array}$ & 96.36 & $\begin{array}{l}458.3 \\
04\end{array}$ & 235.94 & 294.36 \\
\hline $\begin{array}{l}\text { Desv. } \\
\text { Estándar }\end{array}$ & $\begin{array}{l}195.8 \\
9\end{array}$ & $\begin{array}{l}153.9 \\
2\end{array}$ & 86.82 & 76.03 & $\begin{array}{l}187.09 \\
2\end{array}$ & 20.79 & 3.689 & 17.27 & 11.28 & $\begin{array}{l}171.6 \\
53\end{array}$ & 107.99 & 124.69 \\
\hline $\mathbf{P}$ & \multicolumn{2}{|l|}{0.7451} & \multicolumn{2}{|c|}{0.0792} & \multicolumn{2}{|l|}{$0.0177^{*}$} & \multicolumn{2}{|l|}{$0.0234^{*}$} & \multicolumn{2}{|l|}{0.158} & \multicolumn{2}{|l|}{$0.0002^{*}$} \\
\hline
\end{tabular}

Tabla 3. Comparativo de células en los distintos estadios de apoptosis de los grupos de casos y controles del estudio,

\begin{tabular}{|l|l|l|l|}
\hline Variable & $\begin{array}{l}\text { Total de } \\
\text { pacientes }\end{array}$ & $\begin{array}{l}\text { Razón de } \\
\text { momios ajustada }\end{array}$ & $\begin{array}{l}\text { IC de confianza } \\
\text { (95\%) }\end{array}$ \\
\hline Edad & 60 & 1 & $0.855-1.065$ \\
$26-25$ & 38 & & \\
$36-45$ & 8 & 0.50 & \\
\hline Gestas & 54 & & \\
1 & 30 & & \\
2 & 15 & & \\
3 & 2 & 1.2 & \\
4 & 5 & & \\
5 & 12 & & \\
\hline IMC & 76 & & \\
Normal & 16 & & \\
Sobrepeso & 1 & $1.906-1.544$ \\
Obesidad Grado I & \\
Obesidad Grado II & 1 & & \\
Obesidad Grado & 1 & & \\
III & & & \\
\hline Anormalidades & & & \\
nucleares & 32 & & \\
Casos & & & \\
Control & 34 & & \\
\hline
\end{tabular}

Tabla 5.Proporción de presencia de anormalidades nucleares, razón de momios ajustada de acuerdo con las variables de estudio realizado en Hospital General de Pachuca durante el periodo Enero-Junio 2016 
Dentro de los resultados obtenidos en la realización de este estudio, se encuentra relación de anormalidades nucleares, específicamente células binucleadas encontradas en aumento en pacientes con presencia de datos hipertensivos, así mismo se encontró en todas las pacientes con estados hipertensivos de severidad aumento en apoptosis celular, lo que concuerda con Toljic (2017) que asocia el aumento de estrés oxidativo con la muerte celular y daño cromosómico de ADN, propiamente considerado el estado hipertensivo como estrés, desencadenando los mecanismos propios que inducen a liberación de sustancias inflamatorias. La presencia de células binucleadas, podrían ser un marcador importante de falla en la citoquinesis causada por una frecuencia anormalmente elevada de aneuploidía.

Los micronúcleos y las células binucleadas, reflejan el daño al ADN y el efecto genotóxico ocurrido en las células de la capa basal del tejido de las mucosas, los cuales migran hacia la capa epitelial y son detectados en las células exfoliadas en el transcurso de las siguientes tres semanas. El estudio de citoma uno de los ensayos genéticos más utilizado como un indicador de genotoxicidad in vivo, no invasivo y con resultados fehacientes. (Gómez Mireles, et al 2015)

El hallazgo de células apoptóticas en pacientes caso, con diagnóstico de preeclampsia con criterios de severidad, dentro de los resultados; se confirma con los hallazgos propuestos por Rodríguez et, al 2012, en donde se expone de manera detallada la presencia de unas sustancias llamadas microfragmentos de sinciciotrofoblasto (STBM), las cuales alteran el comportamiento de los macrófagos y condicionan un estado proinflamatorio por activación de neutrófilos y alterar el funcionamiento de células endoteliales, todo esto secundario a la hipoxia placentaria, que caracteriza a este estado hipertensivo; generando así un mayor número de células apoptóticas, superando el mecanismo de degradación de estas células, por lo que aumenta su concentración tanto sérica como en distintos tejidos.

Podría considerarse un factor de riesgo la presencia de estas células para desarrollar complicaciones en el feto, ya que la asociación con apoptosis celular en preeclampsia con criterios de severidad se ha asociado con alto riesgo de restricción de crecimiento intrauterino (Sharp et al. 2014); incluso se podría considerar la presencia de estas alteraciones nucleares como factor predictor para desarrollo de preeclampsia 0 de complicaciones fetales, sin embargo se necesita continuar con estudios para corroborarlo.
De acuerdo a Jasovic-Siveska (2011) notificaron que hasta el $85 \%$ de los casos de preeclampsia se presentan en pacientes nulíparas, siendo de 6 a 8 veces más susceptibles que las multíparas. No se ha descrito el mecanismo por el cual ocurre, sin embargo, se presume que durante el primer embarazo se inicia una reacción inmunológica aberrante en la primera exposición a antígenos paternos y fetales extraños a la placenta, que contribuye al desarrollo de preeclampsia.

La unidad fetoplacentaria contiene antígenos paternos que son extraños para la madre huésped, y dichos antígenos serían los responsables de desencadenar todo el proceso inmunológico que provocaría el daño vascular, lo que provocaría el desarrollo de la enfermedad. Durante el primer embarazo se pondría en marcha todo este mecanismo inmunológico y surgiría la preeclampsia; sin embargo, con gestaciones posterior puede desarrollarse el fenómeno de tolerancia inmunológica, que evitará que la enfermedad, siempre que se mantenga el mismo compañero sexual. De modo contrario el efecto protector de la multiparidad se pierde si existe un nuevo compañero sexual. El fenómeno de tolerancia inmunológica disminuye con el tiempo y aproximadamente 10 años después de una primera gestación, la mujer ha perdido la protección que le confiere esta. (Cruz Hernández, et al 2007)

Con los resultados obtenidos en el análisis estadístico se corrobora que la multiparidad es un factor protector contra el desarrollo de preeclampsia coincidiendo con lo reportado en la literatura, comparado con las pacientes nulíparas.

\section{Conclusiones}

El estado hipertensivo, preeclampsia con criterios de severidad, es un estado inflamatorio, que por aumento de radicales libres secundario al estrés oxidativo y otros mediadores de inflamación propician el daño cromosómico; considerando el principal hallazgo el incremento en la frecuencia de células binucleadas como biomarcador de daño a nivel de citocinesis, lo que se corrobora con los resultados de este estudio.

Se confirma el incremento apoptosis celular en pacientes con mayor gravedad de estado hipertensivo, es decir, preeclampsia con criterios de severidad, lo cual se explica de modo que, a mayor estrés oxidativo, mayor liberación de radicales libres que es sometida una paciente; mayor será el daño celular que presente, pudiéndose considerar el hallazgo de estas células un factor predictor en pacientes embarazadas que aún no desarrollan sintomatología hipertensiva, o se encuentran en espera de 
resultados bioquímicos para clasificación de estado hipertensivo; asimismo, en pacientes con factores de riesgo para desarrollar enfermedad hipertensiva (Diabetes mellitus, preeclampsia en embarazo previo, obesidad, edad materna avanzada).

Dado que la fisiopatología de la preeclampsia se relaciona con una alteración entre procesos inflamatorios y disminución de producción o ingesta de antioxidantes, es interesante que, durante el proceso de gestación, la mayoría de las mujeres inician con la ingesta de suplementos multivitamínicos (antioxidantes como complejo B y ácido fólico), estrictamente desde el primer trimestre; lo que podría ayudar a disminuir el daño citogenético en el ADN. Sería importante determinar el nivel de anormalidades nucleares desde el primer trimestre del embarazo y de manera seriada en segundo y tercer trimestre, para evaluar si existe la presencia de anormalidades nucleares, incluso sin aún desarrollar sintomatología hipertensiva; así mismo el seguimiento seriado permitiría evaluar el aumento en las concentraciones $\mathrm{o}$ en la cantidad de anormalidades nucleares, conjuntamente de acuerdo a los resultados valorar si es que existe un desarrollo de estado hipertensivo, o incremento en la severidad de dicho estado. Considerando un factor predictor de preeclampsia en una paciente sin alteraciones hipertensivas.

\section{Referencias}

[1] ACOG Practice Bulletin No. 202. (2019). Obstetrics \& Gynecology, 133(1), e1-e25. https://doi.org/10.1097/aog.0000000000003018

[2] Bolognesi, C., Knasmueller, S., Nersesyan, A., Thomas, P., \& Fenech, M. (2013). The HUMNxl scoring criteria for different cell types and nuclear anomalies in the buccal micronucleus cytome assay - An update and expanded photogallery. Mutation Research/Reviews in $\begin{array}{lll}\text { Mutation } & \text { Research, } & \text { 753(2), }\end{array}$ https://doi.org/10.1016/j.mrrev.2013.07.002

[3] Cruz-Hernández J, Hernández-García P, Yanes-Quesada M, IslasValdez A. (2007). Factores de riesgo de preeclampsia: enfoque inmunoendocrino. Parte I. Rev Cubana Med Gen Integr, 23(4), de http://scielo.sld.cu/scielo.php?script=sci_arttext\&pid=S0864$21252007000400012 \& \operatorname{lng}=$ es\&tlng=es.

[4] Decordier, I., De Bont, K., De Bock, K., Mateuca, R., Roelants, M., Ciardelli, R., Kirsch-Volders, M. (2007). Genetic susceptibility of newborn daughters to oxidative stress. Toxicology Letters, 172(1-2), 68-84. https://doi.org/10.1016/j.toxlet.2007.05.014

[5] Fenech, M., Kirsch-Volders, M., Rossnerova, A., Sram, R., Romm, H., Bolognesi, C., ... Bonassi, S. (2013). HUMN project initiative and review of validation, quality control and prospects for further development of automated micronucleus assays using image cytometry systems. International Journal of Hygiene and Environmental Health, 216(5), 541-552. https://doi.org/10.1016/j.ijheh.2013.01.008.

[6] Furness, D. L. F., Dekker, G. A., Hague, W. M., Khong, T. Y., \& Fenech, M. F. (2010). Increased lymphocyte micronucleus frequency in early pregnancy is associated prospectively with pre-eclampsia and/or intrauterine growth restriction. Mutagenesis, 25(5), 489-498. https://doi.org/10.1093/mutage/geq032
[7] Gómez Carbajal, Luis Martín. (2014). Actualización en la fisiopatología de la preeclampsia: update. Revista Peruana de Ginecología y $\begin{array}{ll}\text { Obstetricia, } & 60(4), \\ \end{array}$ http://www.scielo.org.pe/scielo.php?script=sci_arttext\&pid=S2304$51322014000400008 \& \operatorname{lng}=$ es\&tlng=es

[8] Gómez-Mireles, J., Morales, G., Ortiz, Y., Gómez, B., Guerreo, C., Mendoza, A., Zamora, A. (2015). Disminución del daño nuclear y oxidativo al ADN en pacientes con periodontitis después de la ingesta de ácido fólico. Revista Mexicana de Periodontología, 6(3), 121-128. https://www.medigraphic.com/pdfs/periodontologia/mp2015/mp153c.pdf

[9] Grizzi, F., \& Chiriva-Internati, M. (2007). Human binucleate hepatocytes: Are they a defence during chronic liver diseases? Medical $\begin{array}{lll}\text { Hypotheses, } & 69(2), & 258-261 .\end{array}$ https://doi.org/10.1016/j.mehy.2006.12.029

[10] Hilali, N., Vural, M., Camuzcuoglu, H., Camuzcuoglu, A., \& Aksoy, N. (2013). Increased prolidase activity and oxidative stress in PCOS. $\begin{array}{lll}\text { Clinical } & \text { Endocrinology, } & \text { 79(1), }\end{array}$ https://doi.org/10.1111/cen.12110

[11] Jasovic-Siveska, E., Jasovic, V., \& Stoilova, S. (2011). Previous pregnancy history, parity, maternal age and risk of pregnancy induced hypertension. Bratisl Lek Listy, 112(4), 188-191. Recuperado de http://www.bratislleklisty.sk/2011/11204-07.pdf

[12] Majer, B., Laky, B., Knasmüller, S., \& Kassie, F. (2001). Use of the micronucleus assay with exfoliated epithelial cells as a biomarker for monitoring individuals at elevated risk of genetic damage and in chemoprevention trials. Mutation Research/Reviews in Mutation Research, 489(2-3), 147-172. https://doi.org/10.1016/s13835742(01)00068-0

[13] Secretaria de Salud (2017) Prevención, diagnóstico y tratamiento de la preeclampsia en segundo y tercer nivel de atención Evidencias y Recomendaciones Catálogo Maestro de Guías de Práctica Clínica: IMSS-020-08.

[14] Sharp, A. N., Heazell, A. E. P., Baczyk, D., Dunk, C. E., Lacey, H. A., Jones, C. J. P., ... Crocker, I. P. (2014). Preeclampsia Is Associated with Alterations in the p53-Pathway in Villous Trophoblast. PLoS ONE, 9(1), e87621. https://doi.org/10.1371/journal.pone.0087621

[15] Steegers, E. A., von Dadelszen, P., Duvekot, J. J., \& Pijnenborg, R. (2010). Pre-eclampsia. The Lancet, 376(9741), 631-644. https://doi.org/10.1016/s0140-6736(10)60279-6

[16] Toljic, M., Egic, A., Munjas, J., Karadzov Orlic, N., Milovanovic, Z., Radenkovic, A., Vuceljic, J., Joksic, I. (2017). Increased oxidative stress and cytokinesis-block micronucleus cytome assay parameters in pregnant women with gestational diabetes mellitus and gestational arterial hypertension. Reproductive Toxicology, 71, 55-62. https://doi.org/10.1016/j.reprotox.2017.04.002 